\title{
Analysis and Refinement of the TRC-QSPR Method for Vapor Pressure Prediction
}

\author{
I. Paster $^{1}$, N. Brauner ${ }^{2}$ and M. Shacham ${ }^{1, *}$ \\ ${ }^{1}$ Department of Chemical Engineering, Ben-Gurion University of the Negev, Beer-Sheva 84105, Israel \\ ${ }^{2}$ School of Engineering, Tel-Aviv University, Tel-Aviv 69978, Israel
}

\begin{abstract}
Various aspects associated with the use of the TRC-QSPR method (Shacham et al., Ind. Eng. Chem. Res. 49, 900-912, 2010, Ref. [1]) for the prediction of vapor pressure are investigated using a test set of 12 compounds from the $n$ alkane series. This test set is used to check the consistency of the parameter values of the Wagner and Riedel equations and the resulting vapor pressure values in the full range between the triple point and critical point. Inconsistency has been detected in the parameters of the commonly used version of the Riedel equation as well as the calculated vapor pressure values near the critical point, $T_{R}>0.9$.

Vapor pressures prediction studies are carried out for the cases of interpolation, short and long range extrapolation and using either the acentric factor $(\omega)$, or number of $\mathrm{C}$ atoms $\left(n_{C}\right)$, or the VEAI descriptor in the TRC-QSPR equation. It is concluded that the prediction error is the lowest and within the experimental error limits over the entire temperature range, using the Wagner's equation and $\omega$ within the TRC-QSPR framework. Replacing $\omega$ by $n_{C}$ or by the descriptor VEAI increases the prediction error, however good prediction accuracy is retained in the regions where experimental data are available for the predictive compounds. It is demonstrated that reliable vapor pressure predictions can be obtained using only $n_{C}$ for characterization of the target compound.
\end{abstract}

Keywords: Vapor-pressure prediction, pure component, TRC-QSPR, Wagner equation, Riedel equation.

\section{INTRODUCTION}

Pure component vapor pressure data are essential for phase equilibrium computation, process and product design, in assessing the environmental impact of a chemical compound and in modeling some types of toxicity (Dearden [2]). At present, vapor pressure data are available only for a small fraction of the compounds of interest to the chemical industry. Even if the data are available they may not cover the full temperature range of interest. In product design vapor pressure values may be required for substances that have not been synthesized yet. Thus, prediction of saturated vapor pressure data is often essential.

Current methods used to predict temperature-dependent properties can be classified into "group contribution" methods, methods based on the "corresponding-states principle" (for reviews of these methods see, for example, Poling et al., [3], Godavarty et al., [4] and Velasco et al., [5]) and "asymptotic behavior" correlations (see, for example, Marano and Holder [6]). These methods rely on several other property values, such as normal boiling temperature $\left(T_{b}\right)$, critical temperature $\left(T_{C}\right)$, critical pressure $\left(P_{C}\right)$, and acentric factor $(\omega)$. However, such data for properties may not be available for a target compound, for which the vapor pressure has to be predicted. Moreover, these methods

*Address correspondence to this author at the Department of Chemical Engineering, Ben Gurion University of the Negev, Israel;

Tel: +972-8-64-61481; Fax: +972-8-64-72916;

E-mail: shacham@bgu.ac.il contain adjustable parameters that were fitted to a training set, which may not represent well enough the target compound. A detailed discussion of these issues can be found, for example in Ref. [7].

In recent years, there has been increasing interest in using molecular descriptors integrated into Quantitative Structure Property Relationships (QSPR) for prediction of vapor pressure. However, the great majority of the currently available QSPR models are limited to prediction at a single temperature of $298 \mathrm{~K}$. The exceptions are the methods of Godavarthy [4], which combine their scaled variable reduced coordinates (SVRC) model with neural-network-based QSPRs for representing the nonlinear relations between the SVRC model parameters and molecular descriptors for 1221 molecules. Neural-network QSPR for representing the vapor pressure-temperature behavior of 274 hydrocarbons was used also by Yaffe and Cohen [8].

We are aiming at developing methods for accurate prediction of the vapor pressure-temperature relation for a target compound (the compound for which the property has to be predicted), which are based on minimal data for a few compounds of high level of similarity with the target compound. Such a method (TRC-QSPR method) was suggested by Shacham et al., [1]. In the present work various options for using this method are evaluated and compared and the necessary conditions for obtaining reliable and accurate predictions are discussed. The analysis is carried out for the $n$-alkane homologous series. This series is considered 
a basic reference group of similar compounds for property prediction studies due to the large amount of property data available. Reliable prediction of the properties of high carbon number compounds for the $n$-alkane series can serve as a basis for extending the prediction to other homologous series more complex compounds (see, for example, Willman and Teja, [9]).

\section{METHODOLOGY}

The Two Reference Compound Quantitative Structure Property Relationship (TRC-QSPR) approach has been described in detail and applied successfully for prediction of numerous properties of pure components (Shacham et al., [10], Brauner et al., [11], Shacham et al., [1]). It will be briefly reviewed hereunder.

The TRC-QSPR method is used for predicting temperature (or pressure) dependent properties of a pure target compound, using known property values of two predictive compounds, which are similar to the target. Compounds belonging to the same homologous series of the target compound can be considered "similar". If the identity of similar compounds is not obvious, the Targeted QSPR method Brauner et al., [12] can be used for detecting compounds similar to the target. In the present work the discussion is limited to the case where the target and the predictive compounds belong to the same homologous series.

Application of the TRC-QSPR method requires identification of a molecular descriptor $\zeta^{j}$, which is collinear with the property to be predicted, $y^{\mathrm{p}}$ for the group of compounds similar to the target. The identification of such descriptors is discussed in detail by Brauner et al., [12].

Once such a descriptor has been identified, the temperature or pressure dependent property of a target compound $y_{t}^{p}$ can be predicted (at a particular temperature or pressure) using the following property-property relationship:

$y_{t}^{p}=\frac{\zeta_{2}^{j}-\zeta_{t}^{j}}{\zeta_{2}^{j}-\zeta_{1}^{j}} y_{1}^{p}+\frac{\zeta_{t}^{j}-\zeta_{1}^{j}}{\zeta_{2}^{j}-\zeta_{1}^{j}} y_{2}^{p}$

where $y_{1}^{p}$ and $y_{2}^{p}$ are the property values (at the same reference temperature, or pressure) of two predictive compounds which are similar to the target compound, $\zeta_{1}^{j}$, $\zeta_{2}^{j}$ and $\zeta_{t}^{j}$ are the selected descriptor values for predictive compounds 1 and 2 and the target compound, and $y_{t}^{p}$ is the predicted property value of the target compound.

Shacham et al., [1] proposed two methods for predicting vapor pressure. The first one involves prediction of the saturation temperature $\left(T^{s}\right)$ at a specified vapor pressure. In this case descriptors collinear with the normal boiling temperature ( $T^{S}$ at atmospheric pressure) are used in the property-property relationship. For this case Eq. 1 is rewritten:
$T_{t}^{s}=\frac{\zeta_{2}^{j}-\zeta_{t}^{j}}{\zeta_{2}^{j}-\zeta_{1}^{j}} T_{1}^{\mathrm{s}}+\frac{\zeta_{t}^{j}-\zeta_{1}^{j}}{\zeta_{2}^{j}-\zeta_{1}^{j}} T_{2}^{\mathrm{s}}$

Another option is to predict the logarithm of the reduced vapor pressure, $\ln \left(P_{R}^{s}\right)$ of the target compound at a specified reduced temperature value. Substituting $\ln \left(P_{R}^{s}\right)$ as the predicted property into Eq. 1 yields

$\ln \left(P_{R, t}^{s}\right)=\frac{\zeta_{2}^{j}-\zeta_{t}^{j}}{\zeta_{2}^{j}-\zeta_{1}^{j}} \ln \left(P_{R, 1}^{\mathrm{s}}\right)+\frac{\zeta_{t}^{j}-\zeta_{1}^{j}}{\zeta_{2}^{j}-\zeta_{1}^{j}} \ln \left(P_{R, 2}^{\mathrm{s}}\right)$

where $P_{R, 1}^{s}$ and $P_{R, 2}^{s}$ are the reduced saturation pressures (at a particular reduced temperature of the predictive compounds and $P_{R, t}^{s}$ is the (predicted) reduced saturation pressure of the target compound at $T_{R 0}$. The descriptor $\zeta^{j}$ used in this case must be collinear with $\ln \left(P_{R}^{s}\right)$ at the particular $T_{R 0}$ value. For example, at $T_{R}=0.7$ a descriptor collinear with the acentric factor, $\omega=-\log \left(P_{R}^{s}\right)_{T R=0.7}-1$, can be used. It is assumed the same descriptor is collinear with $\ln \left(P_{R}^{s}\right)$ at other $T_{R}$ values as well. The acentric factor is available for a large number of compounds. It is worth noting that upon using $\omega$ as the descriptor in Eq. 3, the TRCQSPR method reduces to a refined version the traditional "two reference fluid" method, which is discussed in some detail, for example, by Poling et al., [3].

Compared to Eq. 2, Eq. 3 requires more information for predicting the vapor pressure of the target compound (i.e., $P_{c}, T_{c}$ ). However, the application of Eq. (2) requires that the saturation temperatures of the predictive and target compounds correspond to a common range of validity for vapor pressure data [1]. Consequently, difficulties may be encountered in predicting vapor pressure near the critical point or near the triple point. Therefore, in this paper only the TRC-QSPR of Eq. 3 will be considered.

In principle, the TRC-QSPR method can be applied by using experimental vapor pressure values for the predictive compounds. Yet, to obtain vapor pressure values for the predictive compounds at the same specified (reduced) temperature, equations representing the vapor pressure data vs. temperature of the predictive compounds (at least at the vicinity of the specified $T_{R}$ ) are needed. For this aim we use in this work the Riedel equation:

$\ln \left(P_{i}^{s}\right)=A_{i}+\frac{B_{i}}{T}+C_{i} \ln T+D_{i} T^{2}$

and the Wagner equation (as presented by Magoulas and Tassios [13]):

$\ln \left(P_{R}^{s}\right)=\frac{1}{T_{R}}\left(a_{1 i} q+a_{2 i} q^{1.5}+a_{3 i} q^{2.5}+a_{4 i} q^{5}\right) ; \quad q=1-T_{R}$

There are several variants of the Riedel and Wagner equations. The original equation proposed by Riedel [14] uses $T_{R}$ and $P_{R}$ as variables and the exponent on the last term is 6 (instead of the 2 in Eq. 4). Equation 4 was used here as 
the coefficients for a large number of compounds are available for this form (e.g., DIPPR database [15]) and consequently it is more extensively used. In the original Wagner equation [16], the exponents on the last two terms are 3 and 6, respectively (instead of 2.5 and 5). The form of Eq. 5 is used here as the associated coefficients for the compounds of interest were obtained by Ambrose (as cited by Magoulas and Tassios [13]). For evaluation of the accuracy of the TRC-QSPR method, the vapor pressure calculated by either the above vapor pressure models are considered as "true" experimental data.

Equation 3 is used for point-by-point prediction of $P_{R, t}^{s}$ for the target compound at various $T_{R}$ values over the entire liquid phase range. If desired, the predicted vapor pressure values can then be used to fit a vapor pressure model by regression.

Some of the data used in this study are shown in Tables 1 and 2. The compounds used are 12 members of the $n$-alkane homologous series containing between 8 to 30 carbon atoms $\left(n_{C}\right)$. Two compounds: $n$-decane $\left(n_{C}=10\right)$ and $n$-tetradecane $\left(n_{C}=14\right)$ are used as predictive compounds, and the rest of the compounds as target compounds. The critical temperature $\left(T_{C}\right)$, critical pressure $\left(P_{C}\right)$ and the acentric factor $(\omega)$ for all these compounds are listed in Table 1. Two sets of data are included: one from the DIPPR database [15] and the other from Magoulas and Tassios [13]. Observe that there are some differences between the values provided by the different sources. These are however lower than the uncertainties provided in the DIPPR database: the uncertainty on most $T_{C}$ values is $<0.2 \%$, for $n$-eicosane it is
$<1.0 \%$ and for $n$-triacontane $<3.0 \%$. The uncertainty on the $P_{C}$ values of the low $n_{C}$ compounds is $<3 \%$ and it increases up to $<25 \%$ for the high $n_{C}$ compounds.

The Riedel (Eq. 4) constants from the DIPPR database and the Wagner (Eq. 5) coefficients from Magoulas and Tassios [13] are shown in Table 2. For the Riedel equation uncertainty on the calculated vapor pressure values are available. These values are $<1 \%$ or $<3 \%$ for most compounds, $<5 \%$ for $n$-eicosane and $<10 \%$ for $n$ - triacontane. The validity range for the Riedel equation indicated by DIPPR for all the compounds is between the triple point temperature $\left(T_{R} \sim 0.4\right)$ and the critical temperature. Comparing the validity range of the Riedel equation with the range of the available experimental data in the DIPPR database (typically in the range $0.5 \leq T_{R} \leq 0.8$ ) shows that the use of the vapor pressure equations involves extrapolation in the vicinity of the triple and critical points.

To apply the TRC-QSPR method to a target compound with unknown properties, molecular descriptors need to be used to predict $T_{C}, P_{C}$ and $\omega$. To carry out the studies described in this paper, a molecular descriptor database for the $n$-alkane series was prepared. Molecular structures of the various compounds for up to $n_{C}=330$ were drawn using the HyperChem package (Version 7.01, Hyperchem is copyrighted by Hypercube Inc). The Dragon program (version 5.5, DRAGON is copyrighted by TALETE srl, http://www.talete.mi.it, [24]) was used to calculate the descriptors. The limit for molecular size in Dragon 5.5 is 1000 atoms per molecule. This limit dictated the maximal $n_{C}$ $(=330)$ for the molecules used in the study. As 3-D

Table 1. $T_{C}, P_{C}$ and Acentric Factor $(\omega)$ Data from Two Sources, for the Compounds Included in the Study

\begin{tabular}{|c|c|c|c|c|c|c|c|c|c|c|}
\hline & & & \multicolumn{2}{|c|}{$T_{\mathrm{C}}(\mathbf{K})^{*}$} & \multicolumn{2}{|c|}{$P_{C}(\mathbf{M P a})^{*}$} & \multirow[t]{2}{*}{$\omega^{*}$} & \multirow[t]{2}{*}{$T_{C}(\mathbf{K})^{+}$} & \multirow{2}{*}{$\frac{\boldsymbol{P}_{C}}{(\text { bar })^{+}}$} & \multirow[t]{2}{*}{$\omega^{+}$} \\
\hline No. & Compound & $n_{\mathrm{C}}$ & Value & Uncertainty & $P_{C}(\mathrm{MPa})$ & Uncertainty & & & & \\
\hline 2 & $n$-nonane & 9 & 594.6 & $<0.2 \%$ & 2.29 & $<3 \%$ & 0.44346 & 594.9 & 22.9 & 0.443 \\
\hline 5 & $n$-dodecane & 12 & 658 & $<0.2 \%$ & 1.82 & $<10 \%$ & 0.57639 & 658.65 & 18.3 & 0.573 \\
\hline 6 & $n$-tridecane & 13 & 675 & $<0.2 \%$ & 1.68 & $<10 \%$ & 0.6174 & 676 & 17.1 & 0.618 \\
\hline 7 & $n$-tetradecane & 14 & 693 & $<0.2 \%$ & 1.57 & $<25 \%$ & 0.64302 & 693 & 16.1 & 0.654 \\
\hline 11 & $n$-eicosane & 20 & 768 & $<1.0 \%$ & 1.16 & $<25 \%$ & 0.906878 & 769 & 11.6 & 0.891 \\
\hline 12 & $n$-triacontane & 30 & 844 & $<3.0 \%$ & 0.8 & $<25 \%$ & 1.30718 & - & - & - \\
\hline
\end{tabular}

*Source: DIPPR database [15]

${ }^{+}$Source: [13] 
Table 2. Riedel (Eq. 4) and Wagner (Eq. 5) Coefficients from Two Sources, for the Compounds Included in the Study

\begin{tabular}{|c|c|c|c|c|c|c|c|c|c|c|}
\hline \multicolumn{9}{|c|}{ Riedel Equation $^{*}$} & \multicolumn{4}{|c|}{ Wagner Equation $^{+}$} \\
\hline \hline No. & Compound & $\boldsymbol{A}$ & $\boldsymbol{B}$ & $\boldsymbol{C}$ & $\boldsymbol{D}$ & $\boldsymbol{U}$ Ucert. & $\boldsymbol{a}_{1}$ & $\boldsymbol{a}_{2}$ & $\boldsymbol{a}_{3}$ & $\boldsymbol{a}_{4}$ \\
\hline \hline 1 & $n$-octane & 96.084 & -7900.2 & -11.003 & $7.1802 \mathrm{E}-06$ & $<3 \%$ & -8.04937 & 2.03865 & -3.312 & -3.648 \\
\hline 2 & $n$-nonane & 109.35 & -9030.4 & -12.882 & $7.8544 \mathrm{E}-06$ & $<3 \%$ & -8.32886 & 2.25707 & -3.8257 & -3.732 \\
\hline 3 & $n$-decane & 112.73 & -9749.6 & -13.245 & $7.1266 \mathrm{E}-06$ & $<1 \%$ & -8.60643 & 2.44659 & -4.2925 & -3.908 \\
\hline 4 & $n$-undecane & 131 & -11143 & -15.855 & $8.1871 \mathrm{E}-06$ & $<3 \%$ & -8.85079 & 2.60205 & -4.7305 & -4.081 \\
\hline 5 & $n$-dodecane & 137.47 & -11976 & -16.698 & $8.0906 \mathrm{E}-06$ & $<1 \%$ & -9.08593 & 2.77846 & -5.1985 & -4.176 \\
\hline 6 & $n$-tridecane & 137.45 & -12549 & -16.543 & $7.1275 \mathrm{E}-06$ & $<3 \%$ & -9.32959 & 2.89925 & -5.555 & -4.47 \\
\hline 7 & $n$-tetradecane & 140.47 & -13231 & -16.859 & $6.5877 \mathrm{E}-06$ & $<3 \%$ & -9.5447 & 3.06637 & -6.007 & -4.53 \\
\hline 8 & $n$-pentadecane & 135.57 & -13478 & -16.022 & $5.6136 \mathrm{E}-06$ & $<3 \%$ & -9.80239 & 3.29217 & -6.5317 & -4.584 \\
\hline 9 & $n$-hexadecane & 156.06 & -15015 & -18.941 & $6.8172 \mathrm{E}-06$ & $<3 \%$ & -10.03664 & 3.41426 & -6.8627 & -4.863 \\
\hline 10 & $n$-heptadecane & 156.95 & -15557 & -18.966 & $6.4559 \mathrm{E}-06$ & $<3 \%$ & -10.236 & 3.54177 & -7.1898 & -5 \\
\hline 11 & $n$-eicosane & 203.66 & -19441 & -25.525 & $8.8382 \mathrm{E}-06$ & $<5 \%$ & -10.97958 & 4.25588 & -8.9573 & -5.043 \\
\hline 12 & $n$-triacontane & 386.27 & -34581 & -50.953 & $1.6324 \mathrm{E}-05$ & $<10 \%$ & - & - & - & - \\
\hline
\end{tabular}

*Source: DIPPR database [15]

${ }^{+}$Source: [13].

geometry optimization of the structures was not carried out, 3-D descriptors were excluded from the data base.

\section{ANALYSIS OF THE CONSISTENCY OF THE RIEDEL AND WAGNER EQUATIONS}

Usually, vapor pressure equations are evaluated by analyzing their ability to represent the vapor pressure data in the entire range between the triple point and $T_{C}$. The parameters of the vapor pressure equations are obtained by fitting the model to experimental data. However, the set of parameters which adequately represent the data may not be unique, since the various temperature terms in the model are correlated, and consequently their corresponding parameter values are correlated, as well. A more careful evaluation of the vapor pressure model requires the analysis of the consistency of the variation of the equation parameters and the resulting vapor pressure values within the family of similar compounds.

In homologous series, the reduced vapor pressure at a specified reduced temperature varies smoothly with the change of the carbon number, $n_{C}$. Consequently, it can be expected that the change of the equation parameters will also exhibit a smooth variation with $n_{C}$. In Fig. (1) the Wagner equation coefficients (normalized by dividing them by the respective coefficient of the largest absolute value) are plotted versus $n_{C}$. Observe that all coefficients vary monotonically with $n_{C}$, except $a_{4}$, for which there are some small deviations. In fact, the variation of the coefficient values can be represented by low order polynomial of $n_{C}$. On the other hand, the plot of the normalized Riedel equation coefficients (Fig. 2) reveals inconsistent variation due to deviating points $\left(n_{C}=15\right.$, for example) for all the coefficients, with essentially random behavior of the coefficient $D$. Based on our previous study on this subject (Brauner and Shacham [17]) we believe that the high level inconsistency of the Riedel equation parameters is caused by numerical illconditioning, as there are several orders of magnitude difference between the various terms included in Eq. 4. For $n$-pentadecane, for example, $T_{C}=708 \mathrm{~K}$ and the highest temperature for which vapor pressure value is available is $T$ $=577 \mathrm{~K}$. Thus, in Eq. $4(1 / T)=1.76 \times 10^{-3}$, while $T^{2}=$ $3.33 \times 10^{5}$ (i.e., 8 orders of magnitude difference between the smallest and the largest terms). Such a large difference between the terms results in an ill-conditioned regression problem, which may lead to statistically insignificant parameter values, which exhibit inconsistent variation within a group of similar compounds. In comparison, in the case of the Wagner equation, $T_{R}=0.815 ; q=0.185$ and $q^{5}=0.034$, thus the largest difference between the various terms is less than two orders of magnitude. In order to avoid illconditioning it is important to rescale the terms included in the model. It can be expected, for example, that the Riedel equation which is formulated in terms of reduced vapor pressure vs. the reduced temperature, would exhibit a consistent behavior. However, the investigation of this option is outside the scope of the present paper.

The high level of inconsistency of the Riedel equation coefficient values completely rules out the option of developing of a reliable QSPR for predicting directly its coefficients (instead of the point by point prediction of the vapor pressure values). For the Wagner equation, the small inconsistency of the $a_{4}$ values implies that the point-by-point prediction would also result is better prediction accuracy. 
To test the consistency of the calculated vapor pressure values $\left(P^{S}\right)$ in the full range between the triple point and $T_{C}$, the $\ln \left(P_{R}\right)$ values were plotted versus $n_{C}$ for various $T_{R}$ values in this range. One such plot for $T_{R}=0.97$ is shown in Fig. (3). Observe that when the Wagner equation is used for the calculations, $\ln \left(P_{R}\right)$ decreases monotonically from -0.24 at $n_{C}=8$ to -0.3 at $n_{C}=17$. On the other hand, when the Riedel equation is used, the behavior of $\ln \left(P_{R}\right)$ is inconsistent: it starts with the value of -0.24 at $n_{C}=8$, increases to -0.235 at $n_{C}=9$, decreases to -0.25 at $n_{C}=10$, and reaches finally the value of -0.28 at $n_{C}=17$.

Examining the variation of $\ln \left(P_{R}\right)$ versus $n_{C}$ over the entire range of interest shows that the Wagner equation yields consistent $\ln \left(P_{R}\right)$ values from close to the triple point up to the critical point, while with the Riedel equation inconsistent $\ln \left(P_{R}\right)$ values are obtained for $T_{R}>0.9$.

\section{SELECTION OF THE DESCRIPTORS FOR REP- RESENTING THE ACENTRIC FACTOR, $T_{C}$ AND $P_{C}$}

Descriptors that are collinear with the acentric factor for members of the $n$-alkane series in the region where data are available (and do not converge to a constant value for $n_{C} \rightarrow \infty$ ) were considered by Brauner et al., [18]. Two potential candidates were identified: the descriptor $n_{\mathrm{C}}$ and the descriptor VEA1. Using $\omega$ values of $n$-alkanes in the range of $7 \leq n_{C} \leq 17$ the following linear relationship between $n_{C}$ and $\omega$ was obtained:

$$
\omega=0.0761729( \pm 0.0226)+0.040814( \pm 0.00182) \cdot n_{C}
$$

with a correlation coefficient of $R^{2}=0.9965$ and variance of $\rho=7.129 \cdot 10^{-5}$.

The descriptor VEAI is a 2-D eigenvalue-based index and it is defined by the coefficients of the eigenvector associated

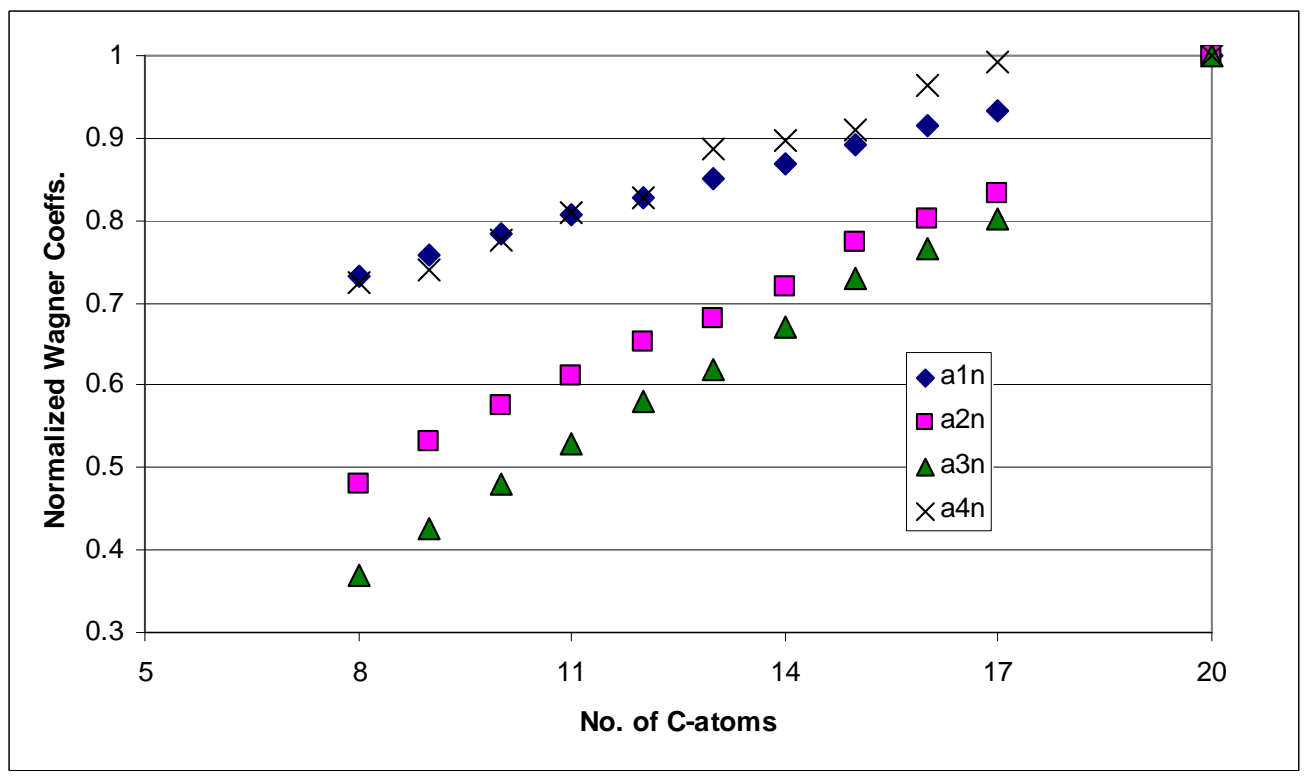

Fig. (1). Plot of normalized Wagner equation coefficients versus $n_{C}$

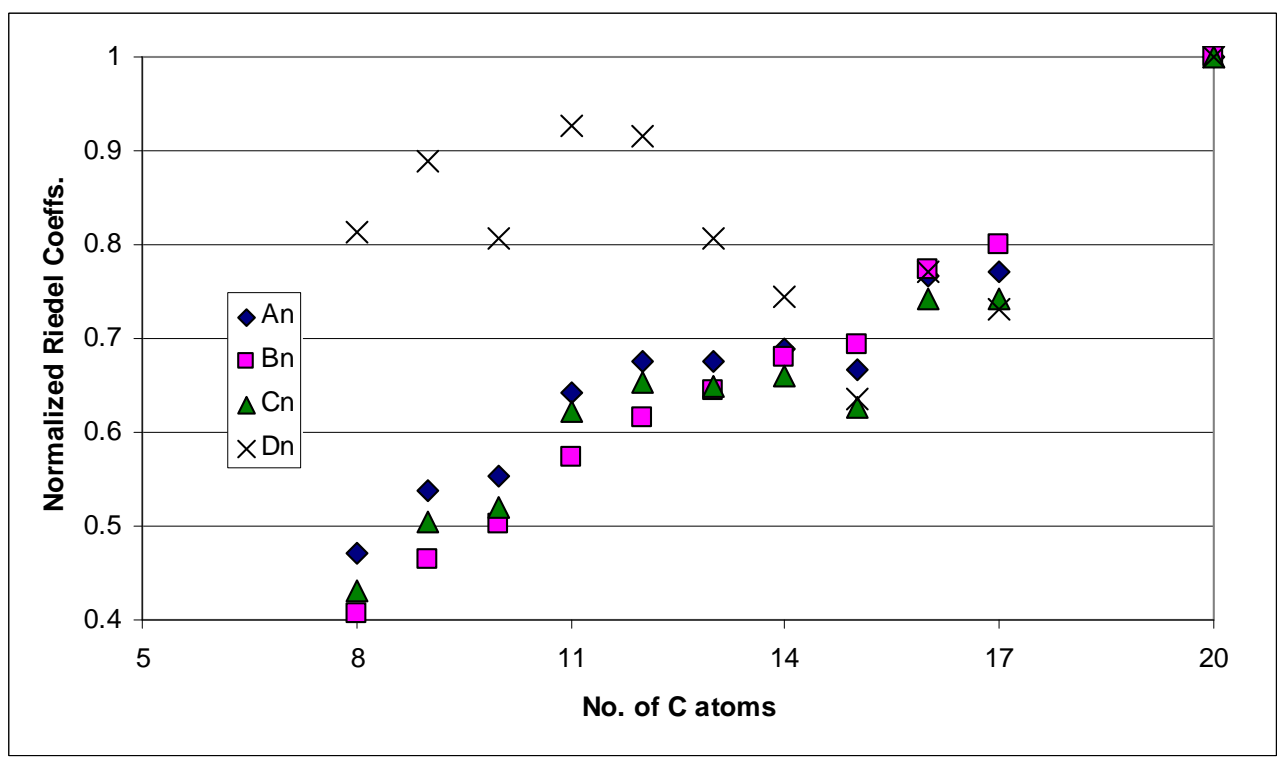

Fig. (2). Plot of normalized Riedel equation coefficients versus $n_{C}$. 


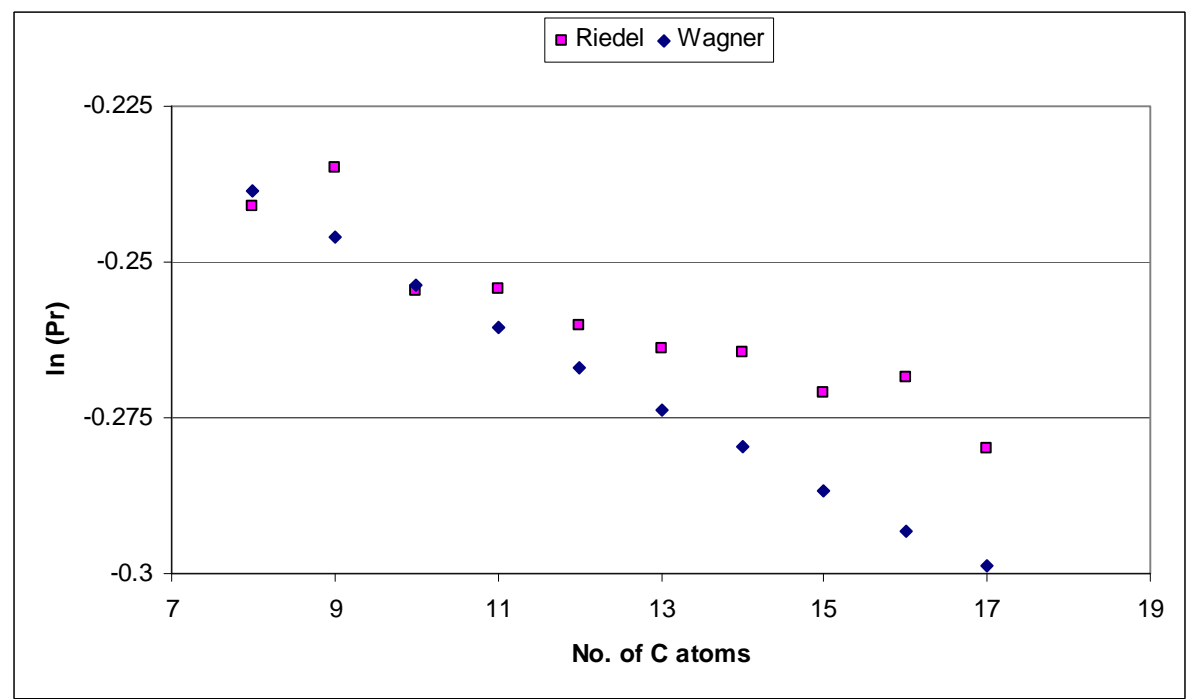

Fig. (3). Variation of calculated values of $\ln \left(P_{R}\right)$ near the critical point (at $T_{R}=0.97$ ) as function of $n_{C}$.

with the largest negative eigenvalue of the adjacency matrix. The values of the VEAI descriptor for the members considered of the $n$-alkane series are shown in Table 3. For large $n_{C}(>20)$, VEAl is highly correlated with $n_{C}^{1 / 2}$ $\left(V E A 1=0.1636238+0.884777 \sqrt{n_{\mathrm{C}}}\right.$, with a correlation coefficient of $\left.R^{2}=0.9999\right)$. Using $\omega$ values of $n$-alkanes in the range of $7 \leq n_{C} \leq 17$ for the training set yields the following linear QSPR:

$\omega=-0.448629( \pm 0.0309)+0.3167022( \pm 0.00958) \cdot V E A 1$

Table 3. VEA1 Descriptor Data for the $n$-alkane Homologous Series

\begin{tabular}{|c|l|c|}
\hline $\boldsymbol{n}_{\boldsymbol{C}}$ & Compound Name & $\begin{array}{c}\text { Descriptor* } \\
\text { VEA1 }\end{array}$ \\
\hline \hline 7 & $n$-heptane & 2.514 \\
\hline 8 & $n$-octane & 2.673 \\
\hline 9 & $n$-nonane & 2.824 \\
\hline 10 & $n$-decane & 2.966 \\
\hline 11 & $n$-undecane & 3.101 \\
\hline 12 & $n$-dodecane & 3.23 \\
\hline 13 & $n$-tridecane & 3.355 \\
\hline 14 & $n$-tetradecane & 3.474 \\
\hline 15 & $n$-pentadecane & 3.59 \\
\hline 16 & $n$-hexadecane & 3.702 \\
\hline 17 & $n$-heptadecane & 3.81 \\
\hline 18 & $n$-octadecane & 3.915 \\
\hline 19 & $n$-nonadecane & 4.018 \\
\hline 20 & $n$-eicosane & 4.118 \\
\hline
\end{tabular}

*For $n_{C}>20$ use the equation $V E A 1=0.1636238+0.884777 \sqrt{n_{\mathrm{C}}}$ with a correlation coefficient of $R^{2}=0.998$ and a variance of $\rho=3.284 \cdot 10^{-5}$.

Due to the high uncertainty (up to $25 \%$ ) of the $P_{C}$ data (which are used for calculation of the acentric factor), a clear conclusion regarding the superiority of one of the two descriptors in representing $\omega$ was not reached by Brauner et al., [18]. In the "Results and Discussion" section, the adequacy of $n_{C}$ and $V E A l$ in representing the acentric factor will be evaluated based on the resulting accuracy of $P^{S}$ predictions.

In Eq. 3 the $P_{R}^{S}$ values are predicted for the target at a particular $T_{R}$ value. To convert these values into $P^{S}$ versus $T$, values of $T_{C}$ and $P_{C}$ of the target compounds are needed. Paster et al., [19] developed single descriptor, linear QSPRs that enable reliable prediction of $T_{C}$ and $P_{C}$ for the $n$-alkane series for medium and high $n_{C}$ values. For the prediction of $T_{C}$, in the range of $n_{C}>5$, the following QSPR was proposed [19]:

$T_{C}(K)=1045.911-591.805 \cdot I V D E$

where the symbol IVDE corresponds to the mean information content of the vertex degree equality. For the $n$ alkane series this descriptor is given by the following function of $n_{C}$ :

$I V D E=-\left[\frac{2}{n_{C}} \log _{2} \frac{2}{n_{C}}+\frac{n_{C}-2}{n_{C}} \log _{2}\left(\frac{n_{C}-2}{n_{C}}\right)\right]$

The descriptor HNar (Narumi's harmonic topological index) is recommended by Paster et al., [19] for predicting $P_{\mathrm{C}}$ in the range of $n_{C}>8$, using the following QSPR:

$P_{\mathrm{C}}(\mathrm{MPa})=12.535-6.261 \cdot H N a r$

where for the $n$-alkane series HNar is given by:

$H N a r=\frac{n_{C}}{2+\frac{n_{C}-2}{2}}$

Thus, both $T_{C}$ and $P_{C}$ can be predicted for the members $\left(n_{C} \sim>8\right)$ of the $n$-alkane series based on $n_{C}$ only. 


\section{RESULTS AND DISCUSSION}

Various options for predicting vapor pressure using the TRC-QSPR method were evaluated. In all the cases $n$-decane $\left(n_{C}=10\right)$ and $n$-tetradecane $\left(n_{C}=14\right)$ were used as the two predictive compounds. The target compounds used for prediction were $n$-octane $\left(n_{C}=8\right.$, extrapolation to lower carbon number), $n$-dodecane ( $n_{C}=12$, interpolation), $n$ heptadecane $\left(n_{C}=17\right.$, extrapolation to higher carbon number) and $n$-eicosane $\left(n_{C}=20\right.$, longer extrapolation to higher carbon number). The Wagner and Riedel equations (with the coefficients shown in Table 2) were used to calculate the reduced vapor pressure values for various $T_{R}$ values in order to obtain $\ln \left(P_{R}\right)_{\text {calc }}$ for the predictive and target compounds. The values of $\ln \left(P_{R}\right)_{\text {pred }}$ for the target compounds were obtained using the TRC-QSPR method (Eq. 3) with either $\omega, n_{C}$ or VEAI as descriptors. The prediction error $\left(\varepsilon_{p}\right)$ was calculated using the equation:

$\varepsilon_{p}=\ln \left(P_{R}\right)_{\text {calc }}-\ln \left(P_{R}\right)_{\text {pred }}$

where $\varepsilon_{p}$ represents the absolute error in $\ln \left(P_{R}^{s}\right)$ and the relative error in $P_{R}^{s}$. The values of $\ln \left(P_{R}\right)_{\text {calc }}$ (for the target compound) were calculated using the Wagner or Riedel equations with the coefficients presented in Table 2. The prediction results are summarized in Figs (4-7). In Fig. (4) the prediction error is plotted versus $T_{R}$ for the case where $\ln \left(P_{R}\right)_{\text {calc }}$ is calculated using the Wagner equations and the Maguolas and Tassios $\omega$ values (Table 1) are used in Eq. (3). The prediction error is the highest near the triple point $\left(T_{R}=\right.$ 0.4 ) and reduces gradually to minimum approaching the critical point. In the range where experimental data are available (usually $\left.0.5 \leq T_{R} \leq 0.8\right) \varepsilon_{p}<2 \%$, which is below the vapor pressure uncertainty limit provided by DIPPR (Table 2). The prediction error is the smallest for the case of interpolation $\left(\varepsilon_{p}<0.31 \%\right)$. For the three cases of extrapolation, the prediction errors are considerably higher. The maximal error depends on the extrapolation distance.
The errors tend to be higher in the case of extrapolation to lower $n_{C}$ compounds.

The plots in Fig. (5) are obtained when the Riedel equation and the DIPPR $\omega$ values (Table 1) are used. The prediction error for interpolation (target $n_{C}=12$ ) is $\varepsilon_{p}<5 \%$. The error distributions for the cases involving extrapolation show minima near $T_{R}=0.7$, and the errors are considerably higher than those obtained when the Wagner equation was used. The maximal error exceeds $40 \%$ near the triple point and $5 \%$ at $T_{R}=0.85$ (for extrapolation to $n_{C}=20$ ). As the Wagner equation yields more accurate predictions, only this equation will be used for rest of the evaluations.

Fig. (6) shows the prediction errors which result when $n_{C}$ is used as the descriptor in Eq. (3). In this case there is a moderate increase in the prediction errors in comparison to the use of $\omega$. When the prediction involves interpolation, the maximal error increases to $\varepsilon_{p}<1.5 \%$. For the three cases of extrapolation, replacing $\omega$ by $n_{C}$ causes the prediction error to increase with error curves of similar shapes. The error is maximal near the triple point $\left(\varepsilon_{p}=9.6 \%\right.$ for extrapolation to $\left.n_{C}=17\right)$ and it decreases gradually toward the critical point. The highest prediction error in the range where data are available is $\varepsilon_{p}=5.6 \%$ at $T_{R}=0.85$.

Similar results were obtained in the case where the descriptor VEAI was used instead of $\omega$ (Fig. 7), except that the prediction errors for long range extrapolation ( to $n_{C}=20$ ) grew very substantially. The maximal error in this case reached $\varepsilon_{p}=36 \%$ close to the triple point. Thus, it seems that for extrapolation to high $n_{C}$ values it is preferable to use $n_{C}$ rather than the descriptor VEA1 in the TRC-QSPR equation.

To further verify the validity of the proposed approach the predictive compounds $n$-decane and $n$-tetradecane were used to predict the vapor pressure curve of $n$-triacontane $\left(n_{C}=30\right)$. The Wagner equation was used for calculating $\ln \left(P_{R}\right)_{\text {calc }}$ values for the predictive compounds and $n_{C}$ was used (as the descriptor) in the TRC-QSPR equation. The $T_{R}$

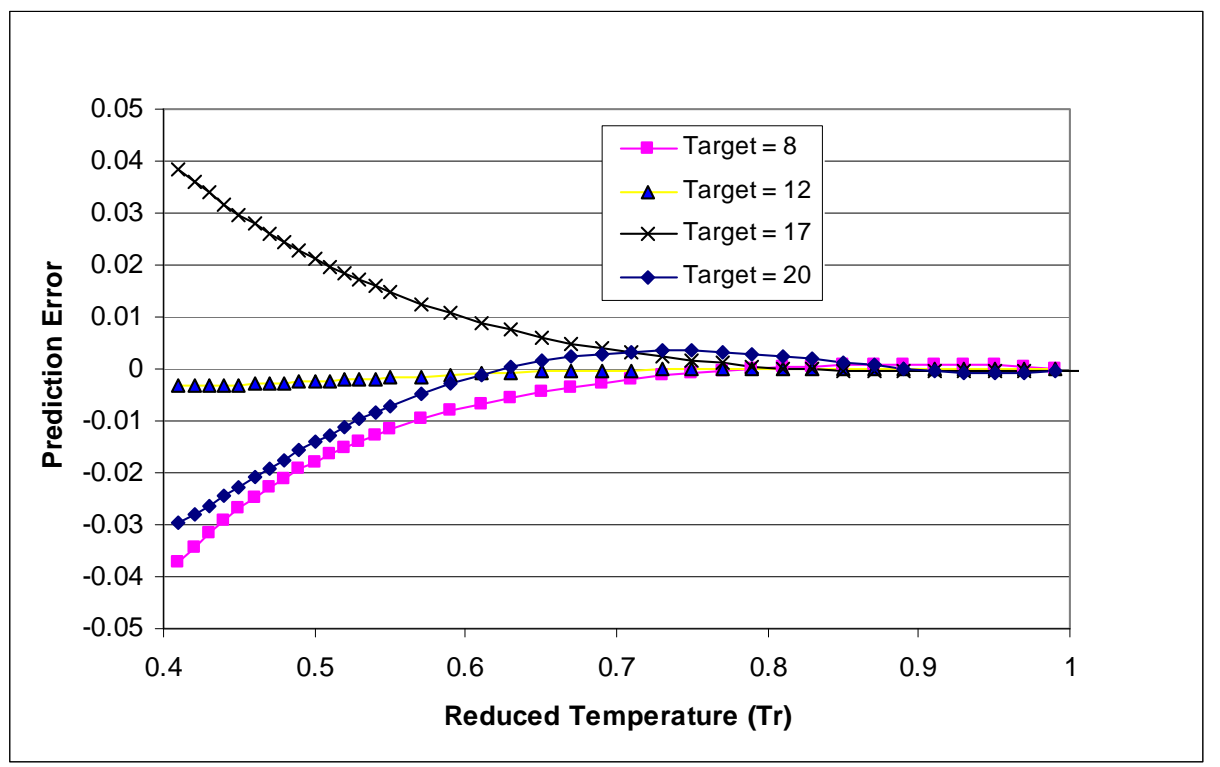

Fig. (4). Prediction of $\ln \left(P_{R}\right)$ using the Wagner equation and Maguolas and Tassios $\omega$ values. 


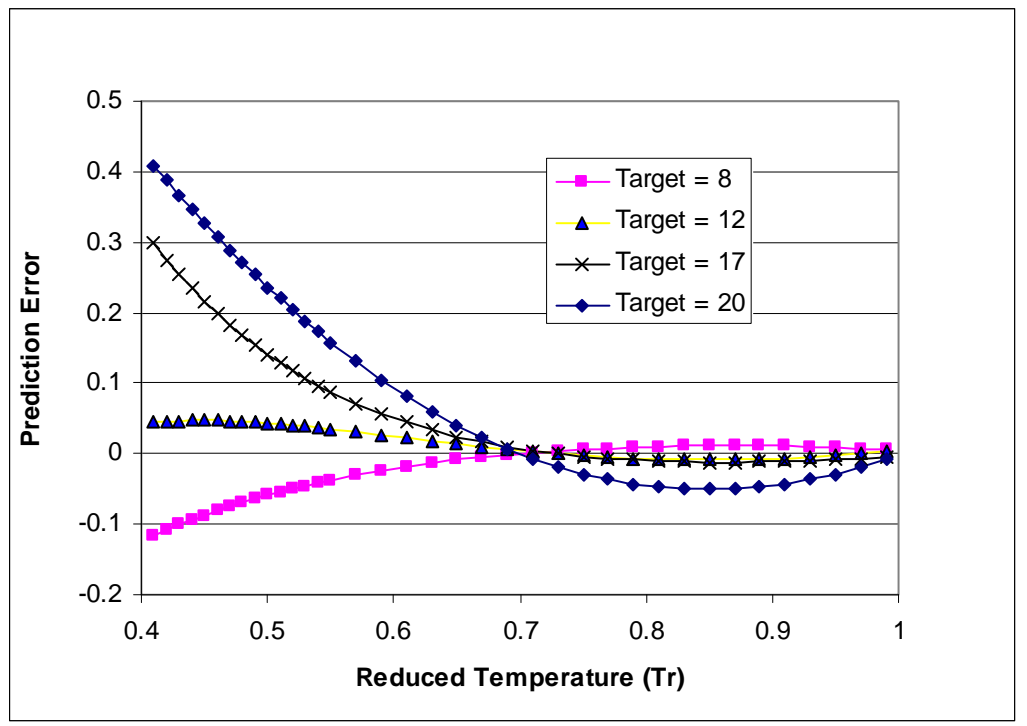

Fig. (5). Prediction of $\ln \left(P_{R}\right)$ using the Riedel equation and the DIPPR $\omega$ values.

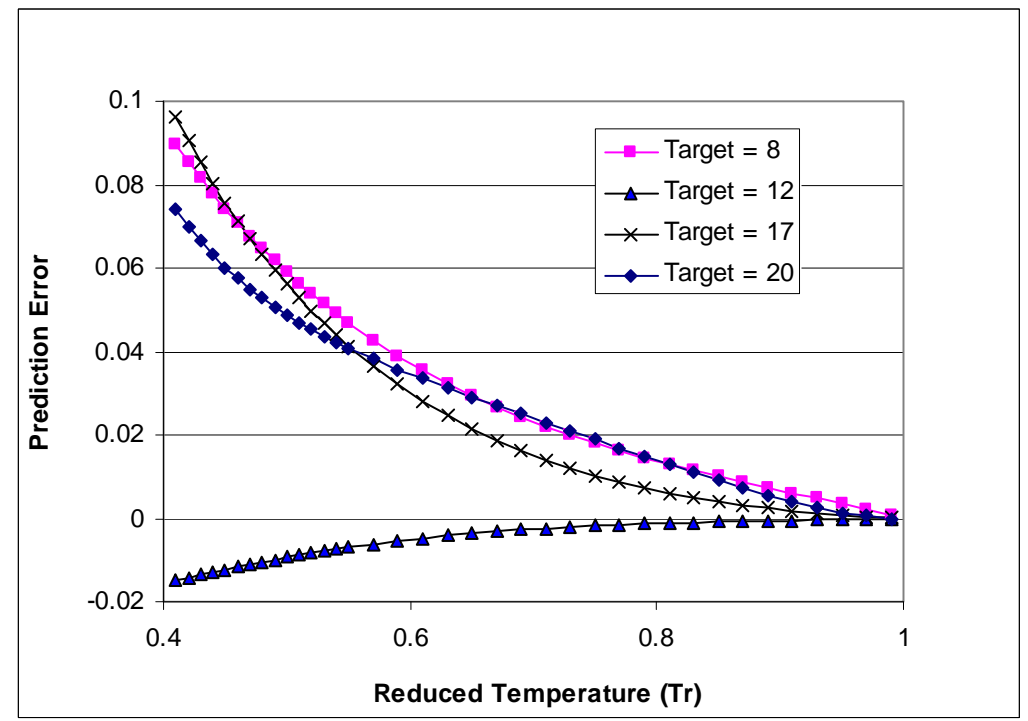

Fig. (6). Prediction of $\ln \left(P_{R}\right)$ using Wagner equation and $n_{C}$ as a predictor.

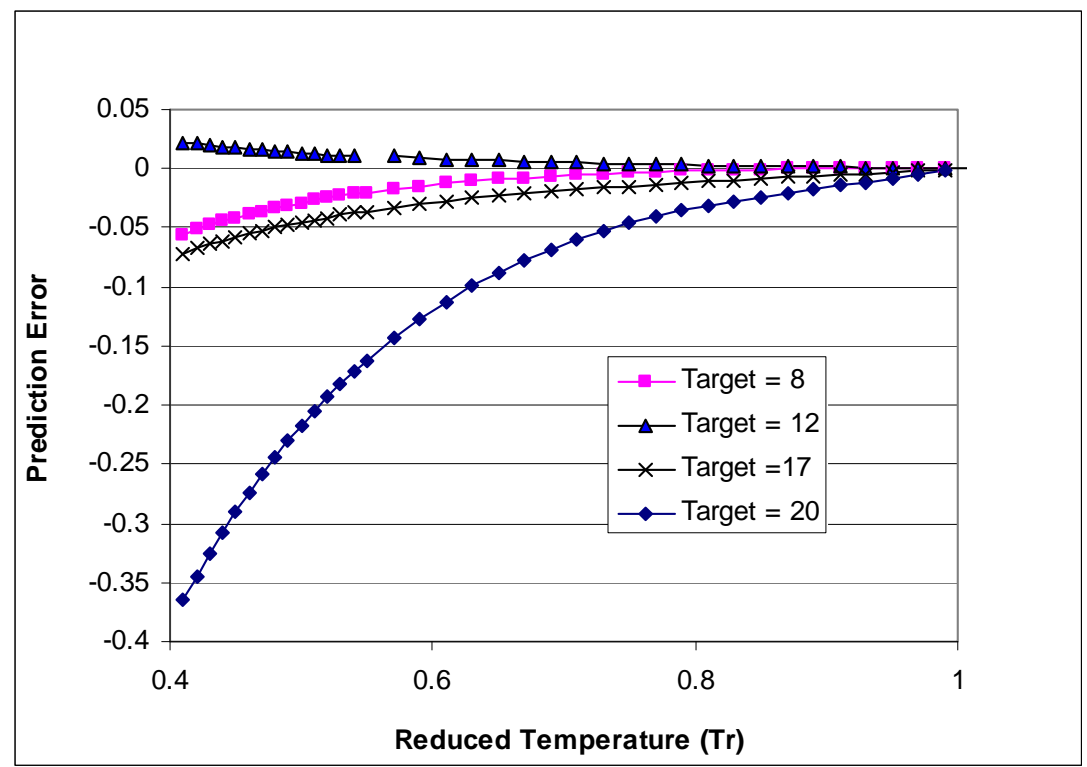

Fig. (7). Prediction of $\ln \left(P_{R}\right)$ using Wagner equation and the VEAl descriptor. 


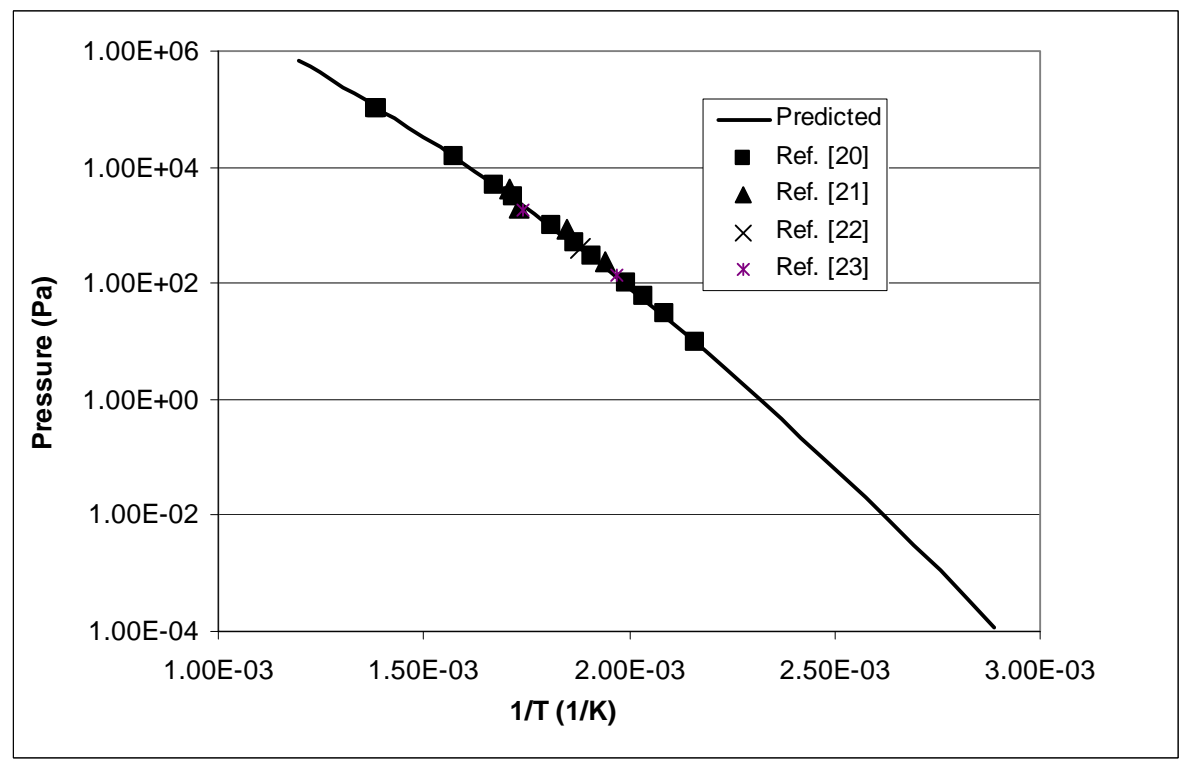

Fig. (8). Predicted vapor pressure curve and reported values for $n$-triacontane. The sources of the experimental data are references [20-23].

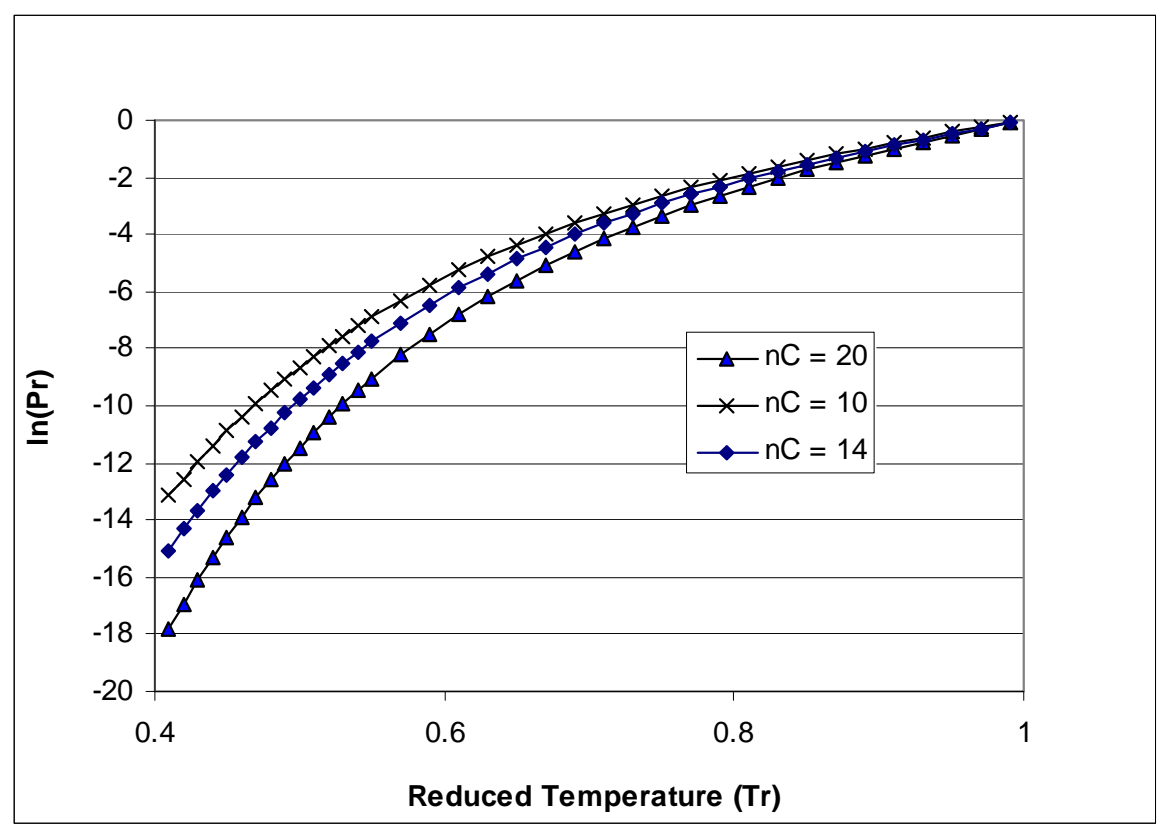

Fig. (9). Variation of $\ln \left(P_{R}\right)$ vs $T_{R}$ for the predictive compounds $\left(n_{C}=10,14\right)$ and the target compound $\left(n_{C}=20\right)$.

and predicted $P_{R}$ values were converted to $T$ and $P^{S}$ using the critical property data of Table 1. Fig. (8) shows that the predicted $P^{S}$ vs $1 / T$ curve compares favorably with the DIPPR database "recommended" data.

The point-by-point predicted values of $P_{R}$ of $n$ triacontane can be used to find the coefficients of the Wagner equation (Eq. 5) by multiple linear regression. The parameter values obtained are $a_{1}=-13.29777 ; a_{2}=$ 5.545463; $a_{3}=-12.86494$ and $a_{4}=-7.018145$. These coefficients are consistent with the Wagner equation coefficients that were provided by Magoulas and Tassios [13] for lower $n_{C} n$-alkanes (Fig. 1).

The substantial increase of the prediction error towards the triple point deserves further explanation. To this aim, observe the plot of $\ln \left(P_{R}\right)$ vs. $T_{R}$ for the predictive $\left(n_{C}=10\right.$, 14) and the target $\left(n_{C}=20\right)$ compounds (Fig. 9). The "distance" between the $\ln \left(P_{R}\right)$ values of the predictive and the target compounds increases monotonically when moving away from the critical point and reaches maximal value toward the triple point. The same behavior can be observed with regard to the prediction error, as shown, for example, in Fig. (6). Thus the larger prediction error is the result of larger extrapolation distances (in cases where the prediction involves extrapolation).

\section{CONCLUSIONS}

A data set of 12 members of the $n$-alkane homologous series containing between 8 to 30 carbon atoms was used to 
investigate various aspects of the TRC-QSPR method for predicting vapor pressure variation with temperature. The analysis was based on the hypothesis that the parameters of the vapor pressure models of the predictive compounds (reference fluids) should be consistent within the family of similar compounds, in addition to the models ability to represent the vapor pressure data in the entire range between the triple point and $T_{C}$. Thus, the test for consistency is another dimension for evaluating the adequacy of different vapor pressure models and for identifying the modifications required for improving the model if necessary.

Analysis of the consistency of the Riedel equation parameters (from the DIPPR database [15]) and the Wagner equation parameters (from [13]) have shown that the Wagner equation coefficients exhibit a consistent variation with $n_{C}$, except for a minor inconsistency in $a_{4}$. On the other hand, the Riedel equation parameters exhibit an inconsistent behavior, with the highest level of inconsistency for the parameter $D$. The analysis of the consistency of the calculated $\ln \left(P_{R}\right)$ values revealed that the Wagner equation yields consistent variation with $n_{C}$ for the entire range between the triple and critical points, while the Riedel equation may yield inconsistent values for $T_{R}>0.9$. The high level of inconsistency of the Riedel equation coefficient values rules out the option of developing reliable QSPRs for predicting directly its coefficients, while the same option may be viable with regard to the coefficient of the Wagner equation.

Using the TRC-QSPR method for the prediction of $\ln \left(P_{R}\right)$ for 4 target compounds (one involving interpolation and three involving extrapolation) has shown that generally, the prediction accuracy significantly deteriorates when the Riedel equation is employed instead of Wagner's equation. With Wagner's equation, the prediction error is the lowest $(<0.35 \%)$ for the case of interpolation and the use of acentric factor $(\omega)$ in the TRC-QSPR equation. Extrapolation, under the same conditions, increases the prediction error, however, still yields $\ln \left(P_{R}\right)$ within experimental error limits except near the triple point. This is a result of the larger extrapolation distances (in terms of $\ln \left(P_{R}\right)$ ) near the triple point.

Replacing $\omega$ by $n_{C}$ or by the descriptor VEAI increases the prediction error considerably, however for interpolation the prediction error still remains below the experimental limit and for extrapolations the predictions are of acceptable accuracy in the region where experimental data are usually available.

Work is currently underway to extend the application of the method to additional homologous series and other groups of similar compounds.

\section{CONFLICT OF INTEREST}

None Declared.

[19] I. Paster, M. Shacham and N. Brauner, " Adjustable QSPRs for Prediction of Properties of Long-chain Substances", AIChE J., vol. 57, no.2, pp. 423-433, 2011.

\section{ACKNOWLEDGEMENT}

[20] Selected Values of Properties of Hydrocarbons and Related Compounds, American Petroleum Institute Research Project 44;

\section{REFERENCES}

[1] M. Shacham, G. St. Cholakov, R. P. Stateva and N. Brauner, "Quantitative Structure-Property Relationships for Prediction of Phase Equilibrium Related Properties", Ind. Eng. Chem. Res., vol. 49, pp. 900-912, 2010.

[2] J. C. Dearden, "Quantitative Structure-Property Relationships for Prediction of Boiling Point, Vapor Pressure, and Melting Point", Environmental Toxicology and Chemistry, vol. 22, no. 8, pp. 16961709, 2003.

[3] B. E. Poling, J. M.Prausnitz and J. P. O'Connel, Properties of Gases and Liquids, $5^{\text {th }}$ Ed., McGraw-Hill: New York, 2001.

[4] S. S. Godavarthy, R. L. Robinson and K. A. M. Gasem, "SVRCQSPR model for predicting saturated vapor pressures of pure fluids", Fluid Phase Equilibria, vol. 246, pp. 39-51, 2006.

[5] S. Velasco, F. L. Román, J. A. White and A. Mulero, "A predictive vapor-pressure equation", J. Chem. Thermodynamics, vol. 40, pp. 789-797, 2008.

[6] J. J. Marano, G. D. Holder, "General Equations for Correlating the Thermo-physical Properties of n-Paraffins, n-Olefins and other Homologous Series. 2. Asymptotic Behavior Correlations for PVT Properties", Ind. Eng. Chem. Res., vol. 36, pp. 1887-1894, 1997.

[7] A. Vetere, "Again the Riedel Equation", Fluid Phase Equilibria, vol. 240, pp. 155-160, 2006.

[8] D. Yaffe and Y. Cohen, "Neural network based temperaturedependent quantitative structure property relations (QSPRs) for predicting vapor pressure of hydrocarbons", J. Chem. Info. Comp. Sci., vol. 41, pp. 463-477, 2001.

[9] B. Willman, A. S. Teja, "Method for the prediction of pure component vapor pressures in the range $1 \mathrm{kPa}$ to the critical pressure", Ind. Eng. Chem. Process Des. Dev., vol. 24, pp. 10331036, 1985.

[10] M. Shacham, N. Brauner, H. Shore and D. Benson-Karhi, "Predicting Temperature-Dependent Properties by Correlations Based on Similarity of Molecular Structures - Application to Liquid Density", Ind. Eng. Chem. Res., vol. 47, pp. 4496-4504, 2008.

[11] N. Brauner, M. Shacham, R. P. Stateva and G. St. Cholakov," Prediction of Phase Equilibrium Related Properties by Correlations Based on Similarity of Molecular Structures", In: J. Jezowski and J. Thullie (Eds), 19th European Symposium on Computer Aided Process Engineering - ESCAPE 19, Krakow, Poland, June 13 - 17, 2009, pp. $69-74$.

[12] N. Brauner, R. P. Stateva, G. St. Cholakov and M. Shacham, "Structurally "Targeted" QSPR Method for Property Prediction", Ind. Eng. Chem. Res, vol. 45, pp. 8430-8437, 2006.

[13] K. Magoulas and D. Tassios, "Thermophysical properties of nalkanes from $\mathrm{C} 1$ to $\mathrm{C} 20$ and their prediction for higher ones", Fluid Phase Equilibria, vol. 56, pp. 119-699, 1990.

[14] L. Riedel, "Neue Dampfdruckformel", Chem. Ing. Tech., vol. 26, pp. 83-89, 1954.

[15] E. L. Rowley, W. V. Wilding, J. L. Oscarson, Y. Yang and N. Zundel, DIPPR Data Compilation of Pure Chemical Properties Design Institute for Physical Properties; Brigham Young University Provo Utah, 2006. http://www.aiche.org/dippr/ (accessed Sept. 2011).

[16] W. Wagner, "New vapor pressure measurements for argon and nitrogen, and a new method for establishing rational vapor pressure equation", Cryogenics, vol. 13, p. 470, 1973.

[17] N. Brauner and M. Shacham, "Role of Range and Precision of the Independent Variable in Regression of Data", AIChE J., vol. 44, no.3, pp. 603-611, 1998.

[18] N. Brauner, I. Paster and M. Shacham, "Linear QSPRs for the Prediction of Acentric Factor and Critical Volume of Long-Chain Substances", paper 672e, Presented at the 10AIChE Annual Substances", paper 672e, Presented at the
Meeting, Salt Lake City, UT, Nov. 7-12, 2010.

None Declared. 
Thermodynamic Research Center, Texas A\&M University, College Station, Texas, 1980-extant. loose-leaf data sheets.

[21] N. E. Wood and F. Francis, "The Boiling Points of Some Higher Aliphatic n-Hydrocarbons"; J. Chem. Soc., vol. 129, p. $1420,1926$.

[22] Catalog Handbook of Fine Chemicals, Aldrich, Milwaukee, WI, 1990.
[23] E. von Sydow, E. Stenhagen, "On the Phase Transitions in Normal Chain Carboxylic Acids with $12 \mathrm{Up}$ To and Including 29 Carbon Atoms Between $30 \mathrm{C}$ and the Melting Point"; Ark. Kemi., vol. 6, pp. 309,1954

[24] Todeschini R, Consonni V, Mauri A, Pavan M. DRAGON User's Manual. Milano, Italy: Talete srl, 2006., http://www.talete.mi.it (accessed Sept. 2011)

(C) Paster et al.; Licensee Bentham Open.

This is an open access article licensed under the terms of the Creative Commons Attribution Non-Commercial License (http://creativecommons.org/licenses/by-nc/3.0/) which permits unrestricted, non-commercial use, distribution and reproduction in any medium, provided the work is properly cited. 